\title{
Laboratory constraints on ice formation, restructuring and desorption
}

\author{
Karin I. Öberg \\ Harvard-Smithsonian Center for Astrophysics \\ 60 Garden St, Cambridge, MA 02138 \\ email: koberg@cfa.harvard.edu
}

\begin{abstract}
Ices form on the surfaces of interstellar and circumstellar dust grains though freezeout of molecules and atoms from the gas-phase followed by chemical reactions. The composition, chemistry, structure and desorption properties of these ices regulate two important aspects of planet formation: the locations of major condensation fronts in protoplanetary disks (i.e. snow lines) and the formation efficiencies of complex organic molecules in astrophysical environments. The latter regulates the availability of prebiotic material on nascent planets. With ALMA it is possible to directly observe both $(\mathrm{CO})$ snowlines and complex organics in protoplanetary disks. The interpretation of these observations requires a detailed understanding of the fundamental ice processes that regulate the build-up, evolution and desorption of icy grain mantles. This proceeding reviews how experiments on thermal $\mathrm{CO}$ and $\mathrm{N}_{2}$ ice desorption, UV photodesorption of $\mathrm{CO}$ ice, and $\mathrm{CO}$ diffusion in $\mathrm{H}_{2} \mathrm{O}$ ice have been used to guide and interpret astrochemical observations of snowlines and complex molecules.
\end{abstract}

Keywords. astrochemistry, astrobiology, molecular processes, methods: laboratory, ISM: molecules

\section{Introduction}

In the cold and dark phases of star and planet formation the surfaces of interstellar dust grains become coated with icy mantles. These ices are built up in molecular clouds through a combination of direct freeze-out of gas-phase molecules (e.g. $\mathrm{CO} \rightarrow \mathrm{CO}_{\mathrm{gr}}$ ) and freeze-out followed by atom addition reactions (e.g. $\mathrm{O}_{\mathrm{gr}}+\mathrm{H}_{\mathrm{gr}} \rightarrow \mathrm{OH}_{\mathrm{gr}} \rightarrow \mathrm{H}_{2} \mathrm{O}_{\mathrm{gr}}$ ). The resulting ice mantle is typically dominated by $\mathrm{H}_{2} \mathrm{O}$ followed by $\mathrm{CO}$ and $\mathrm{CO}_{2}(20-30 \%$ with respect to $\mathrm{H}_{2} \mathrm{O}$ ) and smaller amounts of $\mathrm{CH}_{3} \mathrm{OH}, \mathrm{CH}_{4}$ and $\mathrm{NH}_{3}$ (Öberg et al. 2011a, Boogert et al. 2015). When a cloud core collapses to form a star, some of the icy grains become incorporated into the circumstellar disk that is the formation site of planets. The volatile composition of forming planets depend intimately on the ice and gas composition in the disk (Öberg et al. 2011b), which is set by the desorption energies of the main ice constituents (together with initial conditions and disk chemistry). Major condensation fronts, of e.g. $\mathrm{H}_{2} \mathrm{O}, \mathrm{CO}_{2}, \mathrm{CO}$ and $\mathrm{N}_{2}$, may locally enhance planet formation.

Icy grain mantles are also important from a prebiotic perspective. Ice chemistry is the proposed main cause of the chemical complexity observed toward protostars (Garrod et al. 2008, Herbst \& van Dishoeck 2009). The complex organics that become incorporated into protoplanetary disks and further into planets may seed the origins of life. The abundance and composition of this organic material depend on the efficiency at which simple ice mantles are converted into different kinds of complex organic ices.

Our ability to model both the organic content and the bulk volatile composition of nascent planets then depends fundamentally on our understanding of a small number of ice processes: accretion from the gas-phase, diffusion of atoms, radicals and molecules on top of and inside of ice, reactions when different species encounter one another in the 


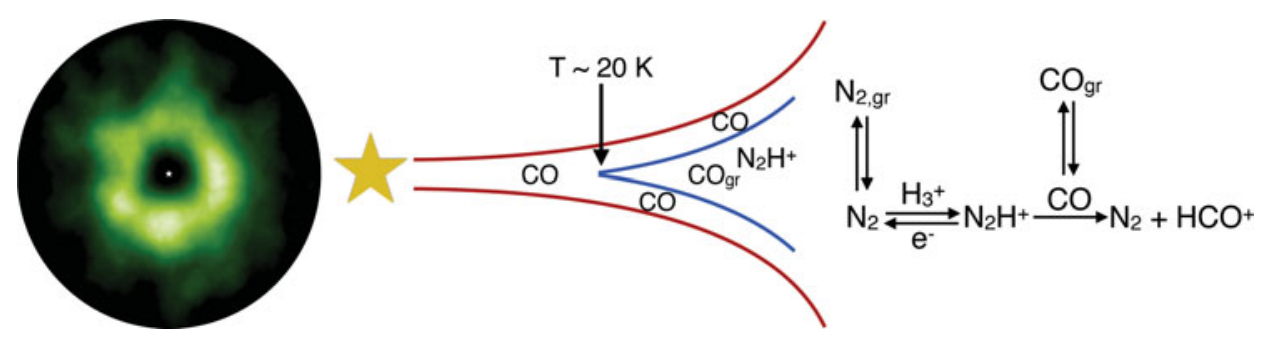

Figure 1. $\mathrm{N}_{2} \mathrm{H}^{+}$observation toward the disk around TW Hya (left, after Qi et al. 2013), and cartoon and reaction diagram summarizing the relation between $\mathrm{CO}$ freeze-out $\left(\mathrm{CO} \rightarrow \mathrm{CO}_{\mathrm{gr}}\right)$ and the appearance of $\mathrm{N}_{2} \mathrm{H}^{+}$in disks. In essence the inner rim of the green $\mathrm{N}_{2} \mathrm{H}^{+}$ring traces the onset of $\mathrm{CO}$ freeze-out in the disk midplane, i.e. the $\mathrm{CO}$ snowline.

ice, and thermal and non-thermal desorption. Quantifying these processes, including their energy barrier heights, requires experiments. This proceeding presents three observations related to disk snowlines and organic chemistry - a $\mathrm{N}_{2} \mathrm{H}^{+}$ring in the disk around young star TW Hya, a double $\mathrm{DCO}^{+}$ring in the disk around young star IM Lup and the presence of complex organics in cold cloud cores - and three ice experiments that aid in interpreting these observations. The focus is on CO, both because it is an excellent model system, and because of its importance for ice organic chemistry and the volatile structure in disks during planet formation.

\section{2. $\mathrm{CO}$ and $\mathrm{N}_{2}$ ice desorption and observations of $\mathrm{CO}$ snowlines}

Snowlines influence several aspects of planet formation, including the efficiency of the initial grain coagulation step and the bulk compositions of forming planets. Observational constraints on snowline locations in disks are therefore important. Based on laboratory experiments on CO desorption energy barriers (Collings et al. 2003), CO snowlines are expected at disk midplane temperatures of $\sim 20 \mathrm{~K}$, which corresponds to disk radii of $\sim 30 \mathrm{AU}$ in disks around young Solar type stars. This is readily resolvable by modern interferometers like the Atacama Large Millimeter and submillimeter Array (ALMA). Direct imaging of snowlines is challenging, however, due to the presence of vertical temperature gradients in disks, which maintains large quantities of $\mathrm{CO}$ in the gas-phase in the disk atmosphere at all disk radii, which can hide loss of $\mathrm{CO}$ emission from the $\mathrm{CO}$ freeze-out zone in the disk midplane (Fig. 1).

A potential solution is to identify a tracer that robustly anti-correlates with $\mathrm{CO}$ gas and therefore traces $\mathrm{CO}$ freeze-out regions. $\mathrm{N}_{2} \mathrm{H}^{+}$is such a tracer as long as $\mathrm{N}_{2}$ freeze-out occurs at (slightly) lower temperatures compared to CO. $\mathrm{N}_{2} \mathrm{H}^{+}$forms through gas-phase reactions between $\mathrm{N}_{2}$ and $\mathrm{H}_{3}^{+}$and is rapidly destroyed by proton transfer to CO, if there is any $\mathrm{CO}$ gas present. If $\mathrm{CO}$ is frozen out and $\mathrm{N}_{2}$ is not, $\mathrm{N}_{2} \mathrm{H}^{+}$can become quite abundant and $\mathrm{N}_{2} \mathrm{H}^{+}$emission should trace the $\mathrm{CO}$ freeze-out zone, i.e. the inner radii of $\mathrm{N}_{2} \mathrm{H}^{+}$ emission should coincide with the $\mathrm{CO}$ snowline. Laboratory experiments have shown that there is a small difference in desorption energy barriers for $\mathrm{CO}$ and $\mathrm{N}_{2}$ in pure ices (Öberg et al. 2005, Bisschop et al. 2006). More recent experiments have demonstrated that this difference persists for water-rich ices (Fayolle et al. in prep.). The utility of $\mathrm{N}_{2} \mathrm{H}^{+}$as a CO snowline tracer was recently demonstrated observationally by the presence of a $\mathrm{N}_{2} \mathrm{H}^{+}$ ring in the disk around the young star TW Hya (Fig. 1), with an inner rim at 30 AU, the expected radius of the CO snowline in this disk (Qi et al. 2013). Snowline locations are thus accessible to observations through interferometric chemical imaging. 

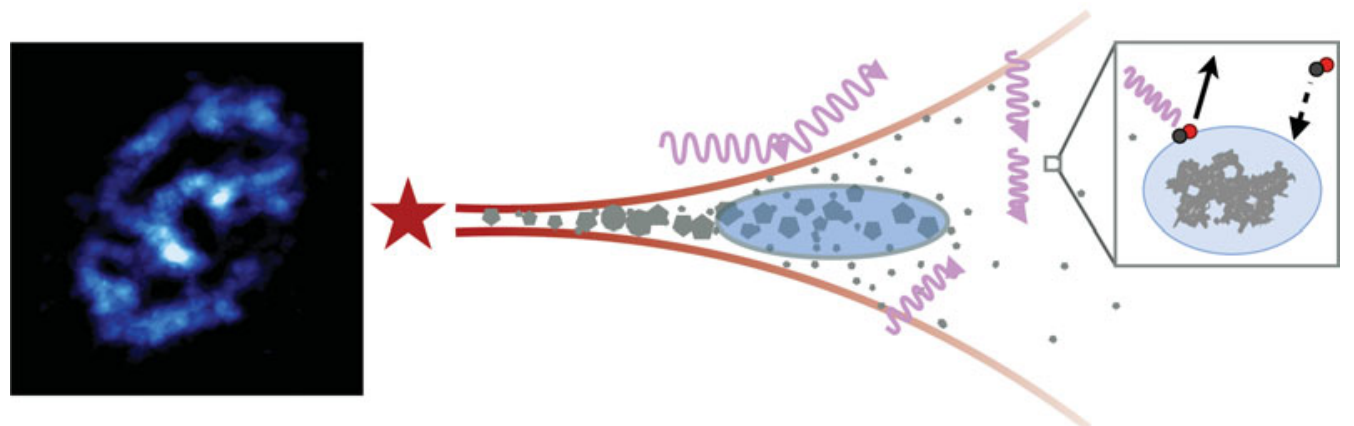

Figure 2. Observation of $\mathrm{DCO}^{+}$in the disk around young star IM Lup (left) and a cartoon illustrating the complete freeze-out zone of $\mathrm{CO}$ in a disk (light blue oval), limited by thermal desorption of $\mathrm{CO}$ inwards, and non-thermal desorption in the outer disk. $\mathrm{DCO}^{+}$is expected to be the most abundant just interior to the inner $\mathrm{CO}$ snowline and in the non-thermal desorption region, where the two ingredients of efficient $\mathrm{DCO}^{+}$formation - low temperatures and $\mathrm{CO}$ gas - are both present.

\section{3. $\mathrm{CO}$ ice photodesorption and a $\mathrm{DCO}^{+}$double ring in a disk}

In addition to the thermal desorption described in the previous section, ices can desorb non-thermally through interactions with UV, electrons, and cosmic rays, and through the release of chemical energy. In the dense, inner regions of protoplanetary disks, the disk mid planes are efficiently shielded from all external radiation. The division of molecules between ice and gas is therefore mainly set by gas adsorption onto grains and thermal desorption of ice, regulating e.g. the location of the CO snowline (Fig. 1). In the absence of non-thermal desorption, almost no $\mathrm{CO}$ is expected in the midplane exterior to the $\mathrm{CO}$ snowline. Further out yet in the disk some the gas can be partially repopulated by CO through a combination of low adsorption rate (caused by the low densities found in the outer regions of disks) and the activation of non-thermal desorption pathways, such as UV photodesorption of ice. The latter can occur if 1) the dust column becomes to small to efficiently shield the disk midplane from external radiation, and 2) UV photodesorption is an efficient process.

UV photodesorption of ices have been explored extensively through laboratory experiments (Öberg et al. 2009a, Munoz Caro et al. 2010, Fayolle et al. 2011), and the efficiencies are high, at least $10^{-3}$ per incident UV photon. These high desorption efficiencies imply that in the outer tenuous disk, UV photodesorption could partially repopulate the disk midplane with $\mathrm{CO}$ gas . The effects of ice photodesorption (or a related non-thermal desorption process) are manifest in the disk of IM Lup. The disk presents a set of concentric $\mathrm{DCO}^{+}$rings (Öberg et al. 2015), where the outer disk can be attributed to $\mathrm{DCO}^{+}$ formation following desorption of (some) CO back into the gas-phase at large disk radii. Based on our understanding of isotopic fractionation chemistry and CO desorption we can interpret the two concentric $\mathrm{DCO}^{+}$rings as marking 4 distinct disk regions: an inner disk region where $\mathrm{CO}$ is in the gas-phase, but where it is too warm for efficient deuterium chemistry (inner hole), a region close to the $\mathrm{CO}$ snowline where $\mathrm{CO}$ is still in the gas phase and temperatures are sufficiently low for $\mathrm{DCO}^{+}$to be enhanced (the inner $\mathrm{DCO}^{+}$ring), a $\mathrm{CO}$ freeze-out region where both thermal and non-thermal desorption are very slow and all CO based chemistry is turned off, and an outer disk region where CO photodesorption becomes efficient and $\mathrm{DCO}^{+}$therefore reappears (the second ring). 


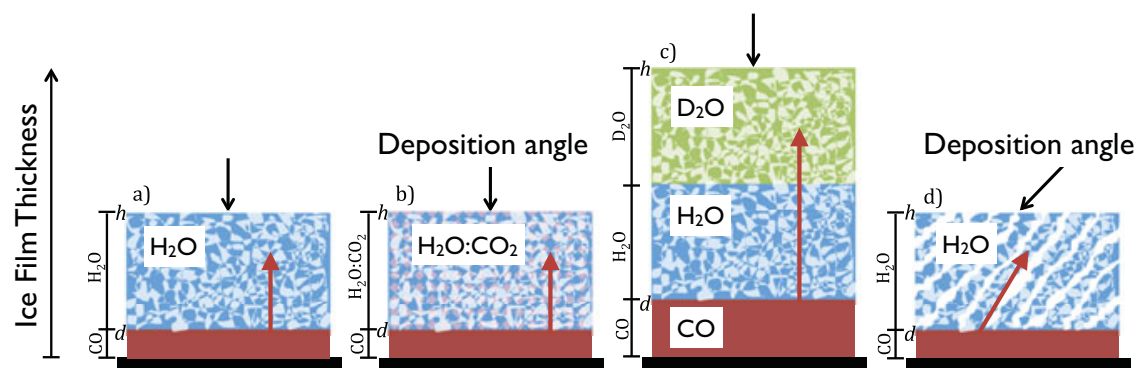

Figure 3. Cartoon illustrating CO diffusion experiments by Lauck et al. (2015), where CO diffusion into water ice was measured spectroscopically employing initially layered $\mathrm{CO}$ and $\mathrm{H}_{2} \mathrm{O}$ ices of different ice thicknesses, porosities and isotopic compositions.

\section{CO ice diffusion and a cold complex organic ice chemistry in space}

A puzzle in astrochemistry is the presence of complex organic molecules in cold molecular cloud cores (Öberg et al. 2010, Bacmann et al. 2012, Cernicharo et al. 2012). In these regions the commonly invoked interstellar pathway for forming complex organics - photodissociation of $\mathrm{CH}_{3} \mathrm{OH}$ and other ice molecules into radicals followed by radical diffusion and reactions - seem to fail. With the currently used prescriptions for diffusion in ices, the icy grain mantles in these cores are simply too cold for radical diffusion (e.g. Garrod et al. 2008). There are very few measurements of diffusion of radicals and molecules in ices, however, and it is therefore unclear whether existing assumptions on diffusion barriers are realistic. A number of recent studies have attempted to measure $\mathrm{CO}$ diffusion in $\mathrm{H}_{2} \mathrm{O}$ ice to test these assumptions (e.g. Karssemeijer et al. 2014, Lauck et al. 2015). Our experimental approach is summarized in Fig. 3. The studies find consistently lower diffusion barriers than expected for $\mathrm{CO}$ in $\mathrm{H}_{2} \mathrm{O}$ ice; we find a diffusion to desorption energy ratio that is $\sim 0.15$, which can be compared to commonly assumed ratios of $0.3-0.7$ (Lauck et al. 2015). This suggests that molecules and radicals can be mobile in ices at lower temperatures than previously assumed, perhaps explaining the existence of complex organics molecules in cold interstellar environments.

\section{References}

Bacmann, A, Taquet, V, Faure, A, et al. 2012, A\&A, 541, 12

Bisschop, S. E. and Fraser, H. J., Öberg, K. I. et al. 2006, A\&\&A, 449, 1297

Cernicharo, J, Marcelino, N, \& Roueff, E, 2012, A\&A, 759, L43

Collings, M. P., Dever, J. W., \& Fraser, H. J. 2003, ApJ, 583, 1058

Fayolle, E. C., Bertin, M., \& Romanzin, C. 2011, ApJL, 739, L36

Garrod R. T., Weaver S. L. W., \& Herbst E. 2008, ApJ, 682, 283

Herbst, E., \& van Dishoeck, E. F. 2009, ARA\&A, 47, 427

Karssemeijer, L. J, Ioppolo, S, \& van Hemert, M. C, 2014, A\&A, 781, 16

Lauck, T., Karssemeijer, L., Shulenberger, K. et al. 2015, ApJ, 801, 118

Munoz Caro, G. M, Jimenez-Escobar, A, \& Martin-Gago, J. A, 2010, A\&A, 522, 108

Öberg, K. I., van Broekhuizen, F., Fraser, H. J. et al. 2005 ApJL, 621, L33

Öberg, K. I., van Dishoeck, E. F., \& Linnartz, H. 2009 A $\& A, 496,281$

Öberg K. I., Boogert A. C. A., Pontoppidan K. M. et al. 2011 ApJ, 740, 109

Öberg, K. I., Murray-Clay, R., \& Bergin, E. A. 2011 ApJL, 743, L16

Öberg, K. I., Furuya, K., \& Loomis, R. 2015 ApJ, 810, 112

Qi, C., Öberg, K. I., Wilner, D. J. et al. 2013, Science, 341, 630 